\title{
HUVECs-CONDITIONED MEDIUM HAS A BETTER POTENTIAL TO STIMULATE DIFFERENTIATION OF DENTAL PULP STROMAL CELLS TOWARD AN OSTEOBLASTIC LINEAGE
}

\author{
Lisa R. Amir, Nurulia Januarti, Raden R. Septiana \\ Department of Oral Biology, Faculty of Dentistry, Universitas Indonesia, Indonesia \\ Undergraduate Program, Faculty of Dentistry, Universitas Indonesia, Indonesia
}

\begin{abstract}
INTRODUCTION: One of the biggest challenges in tissue engineering technique is adequate vascularization to prevent tissue necrosis. This problem often arises in large bone defect reconstruction. During osteogenesis, vascularization involves cross-talk regulation between endothelial and osteoblast cells. It was the foundation of this study.

ОвJеCTIVEs: To evaluate the effect of co-culture and conditioned medium of human umbilical vein endothelial cells (HUVECs) on the expression of collagen I, fibronectin proteins and alkaline phosphates (ALP) activity released by dental pulp cells (DPSCs) on 3D scaffold composed of hydroxyapatite/tricalcium phosphate/chitosan (HA/TCP/chitosan).

MATERIAL AND METHODS: The three main research group were (1) DPSCs co-cultured with various ratios of HUVECs, (2) DPSCs cultured with various concentrations of HUVECs-conditioned medium (CM), (3) DPSCs cultured with normal medium as the control. Culture media were collected at day 3, 5, 7 to examine the collagen type 1, fibronection protein and ALP activity levels.

RESULTS: DPSCs-HUVECs indirect co-culture increased the protein expression of collagen I and fibronectin. However, the upregulation was higher when DPSCs were cultured with HUVECs CM. ALP was not changed in co-culture systems while it increased in the HUVECs CM group. The increase in three different markers tested in HUVECs CM compared to the co-culture system might be explained by the presence of signaling molecules in the HUVECs CM that stimulate DPSCs. Fibronectin expression was necessary for DPSCs' attachment to the 3D scaffold, while collagen I and ALP expressions might indicate the differentiation of DPSCs to osteoblasts.

ConcLusions: HUVECs-conditioned medium has a better potential to be used to stimulate the differentiation of dental pulp cells towards an osteoblastic lineage, the condition that is necessary for the success of bone tissue engineering.
\end{abstract}

KEY WORDS: endothelial cells, dental pulp cells, scaffold, calcium phosphate, chitosan.

J Stoma 2018; 71, 6: 466-471

DOI: https://doi.org/10.5114/jos.2018.85561

\section{JOURNAL OF STOMATOLOGY CZASOPISMO STOMATOLOGICZNE

Address for Correspondence: Lisa R. Amir, Department of Oral Biology, Faculty of Dentistry, University of Indonesia, Salemba Raya No.4, Jakarta Pusat, Indonesia, phone: 021-31930355, fax: 021-31930343, e-mail: lisa.amir@ui.ac.id 


\section{INTRODUCTION}

Bone is one of the highly vascularized tissues and the development of vascularization is critical for bone homeostasis and regeneration. Blood vessels mediate the transport of circulation cells, oxygen, nutrient and angiogenic molecules providing paracrine signals that promote the growth and differentiation of different tissues including bone [1]. Endothelial cells that reside in the blood vessel walls form new blood capillaries by the sprouting of existing vessels in the angiogenesis process and there is intimate communication between endothelial cells and bone cells to maintain the skeletal integrity $[2,3]$. Cross-talk regulation from functional interaction between endothelial cells and osteoblasts during osteogenesis has been previously reported [2-6]. During intra-membranous bone regeneration, osteoblasts sense oxygen tension during the initial inflammatory phase of the healing process and respond to hypoxic conditions by activating the hypoxia-inducible factor $\alpha$ (HIF- $\alpha$ ) pathway that promotes endothelial migration and proliferation [4]. Vascular endothelial growth factor (VEGF) is a key regulator for angiogenesis and it is critical for both bone development and regeneration. VEGF acts both on endothelial cells (ECs) to promote EC migration and proliferation and on osteoblast migration and proliferation though the regulation of osteogenic growth factors $[5,6]$.

In bone tissue engineering, one of the biggest challenges is to have adequate vascularization so as to prevent tissue necrosis. The maintenance of cell viability in the core of the cell-tissue construct depends on the invasion of existing blood vessels though angiogenesis [7]. Poor blood supply results in cell death due to lack of oxygen and nutrients and other soluble factors that are necessary for osteoblasts' proliferation and differentiation. It has been reported that oxygen diffusion to the surrounding tissue is limited to $150 \mu \mathrm{m}$ from the adjacent capillary vessels and the majority of cells located further away from the blood vessels will experience hypoxia [7]. The survival of cells and the scaffold construct therefore rely on the formation of an endogenous capillary network in response to angiogenic factors released by the hypoxic cells and the growth is approximately $100 \mathrm{~nm}$ per day [7]. To accelerate the vascularization of the cell-scaffold construct following in vivo implantation several approaches have been introduced such as incorporating endothelial cells to initiate the angiogenesis process [8-11]. Endothelial cells in a 3D scaffold could be used for large tissue reconstruction and transplanted in vivo. This would not only solve the problem of nutrient and oxygen diffusion to the inner part of the constructed bone tissue, but simultaneously it could stimulate osteogenesis through endothelial cells. As such, incorporating simultaneously multiple strategies of angiogenesis has been proposed to improve the success of bone tissue engineering [8-11].
Oral tissue origin mesenchymal stromal cells (MSCs) such as dental pulp stromal cells (DPSCs) have a great clinical potential and are relatively easy to harvest. One of the earliest reports of DPSCs demonstrated higher numbers of colony-forming cells and more bromodeoxyuridine positive cells than bone marrow derived MSC (BM-MSCs), indicating a larger clonogenic MSC population in DPSCs than in BM-MSCs [12]. Previous studies have reported the interaction of endothelial cells (ECs) with oral tissue origin mesenchymal stromal cells (MSCs) for odontogenesis and osteogenesis [13-16]. ECs' secretory product endothelin-1 (ET-1) influenced the differentiation of DPSCs whereby a higher level of odontoblastic differentiation-associated genes was observed [13]. The interaction between these two cells occurs via direct cell-cell contract and paracrine signaling. Other studies have reported a three-dimensional (3D) co-culture model in hydrogel; more extracellular matrix was deposited, and higher levels of vascularization and mineralization in vivo were seen than in the DPSCs monocultures [14]. Although a number of studies have reported interaction of ECs and DPSCs in direct and indirect co-culture models, the effect of EC-conditioned medium on DPSCs proliferation and differentiation is still unclear. EC-conditioned medium is known to contain numerous EC secreted proteins that could promote DPSCs differentiation [17]. In the present study, we aimed to evaluate the effect of EC-conditioned medium on DPSCs differentiation and compared the results with the effect of direct and indirect EC-DPSCs co-culture.

\section{MATERIAL AND METHODS}

\section{MATERIAL}

DMEM, fetal bovine serum (FBS), penicillin, streptomycin, fungizone, collagenase I, PBS, M200 medium supplemented with LSGS kit, PBS, were purchased were from Gibco, California, USA. CD71, CD90, CD45 antibodies, CD31, V-CAM were from BD Sciences. Dispase from Roche, Indianapolis, USA. Glucose (Sigma, St Louis, USA). Quantichrom ${ }^{\mathrm{TM}}$ Alkaline Phosphatase Assay Kit were from BioAssay Systems, USA. Neutravidin HRP were from ThermoFisher Scientific, USA. Bradford total protein assay (Bio-Rad Protein Assay Kit, Bio-Rad, USA). HA/TCP/chitosan scaffold were manufactured in National Nuclear Energy Agancy of Indonesia, Jakarta, Indonesia. Well-plate, culture flasks were from NUNC, Denmark. Trans-well PET membrane, $8.0 \mu \mathrm{M}$ were form corning USA.

\section{DENTAL PULP STROMAL CELLS ISOLATION}

The study obtained ethical approval from the Dental Research Ethics Committee Faculty of Dentistry Universitas Indonesia. Dental pulp tissues were harvested from 

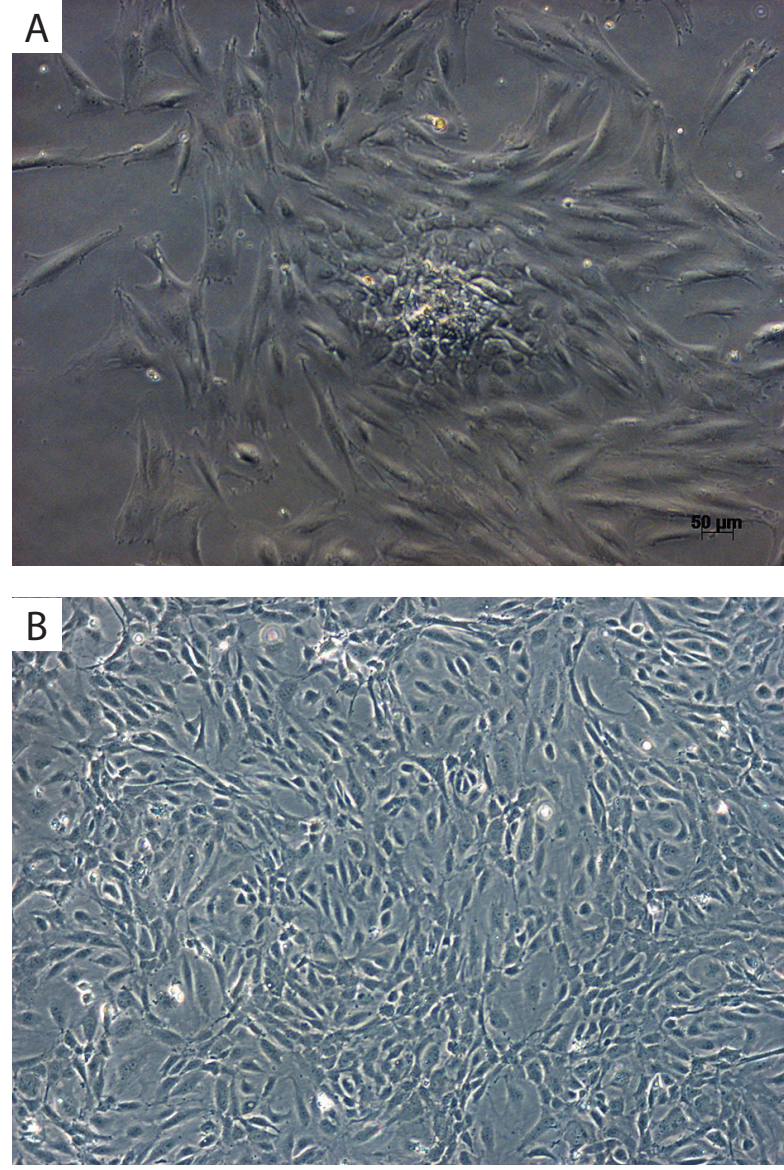

FIGURE 1. Dental pulp stromal cells (DPSCs) and human umbilical vein endothelial cells (HUVECs). A) DPSCs showed their ability to form colony forming units (> 50 cells). B) HUVECs characterized by their morphological structure

three impacted teeth as previously reported [18]. Briefly, the teeth were crushed with a mortar and pestle, removed from the root canal by an extirpation needle and finely chopped with a sterile scalpel. The cells were dissociated from dental pulp tissue by enzymatic degradation with $4 \mathrm{mg} / \mathrm{ml}$ collagenase I (Gibco, California, USA) and $3 \mathrm{mg} / \mathrm{ml}$ dispase (Roche, Indianapolis, USA) in a $15 \mathrm{ml}$ tube (BD Falcon, New Jersey, USA) at $37^{\circ} \mathrm{C}$ under $5 \% \mathrm{CO}_{2}$ in an incubator for $1 \mathrm{~h}$. The process was terminated by adding complete DMEM medium supplemented with $20 \%$ FBS, $100 \mathrm{U} / \mathrm{ml}$ penicillin, $100 \mu \mathrm{g} / \mathrm{ml}$ streptomycin and $1.25 \mu \mathrm{g} / \mathrm{ml}$ Fungizone (Gibco, California, USA). The dental pulp cells were then cultured in a 6-well plate (Nunc, Roskilde, Denmark) until they reached about $90 \%$ confluency for approximately 14 days. $10^{7}$ cells were prepared for cell sorting by fluorescence-activated cell sorting (FACS) in a FACSAria (BD Biosciences, USA) by using CD71, CD90, and CD45 antibodies (BD Biosciences). CD71and CD90-positive cells and CD45-negative cells indicated the presence of DPSCs.

\section{HUMAN UMBILICAL VEIN ENDOTHELIAL CELLS}

\section{ISOLATION}

Endothelial cells were isolated from umbilical cord (human umbilical vein endothelial cells - HUVECs). Umbilical cords were obtained during breech deliveries with cesarean section at Budi Kemuliaan Maternity Hospital, Jakarta, Indonesia. All patients have signed informed consent.

Cord buffer transport medium containing $2 \mathrm{~g} / \mathrm{ml}$ glucose (Sigma, St. Louis, USA) in PBS with $1 \%$ penicillinstreptomycin was used to store umbilical cords of approximately $15 \mathrm{~cm}$. HUVECs isolation was performed within $4 \mathrm{~h}$ of umbilical cord collection. Umbilical veins were washed with sterile PBS with 2 cannulae fixed in both cord extremities to remove the remaining blood clot.

HUVECs cells were separated from the blood vessel wall by injecting $0.2 \%$ collagenase I (Gibco, California, USA) into the umbilical vein and incubated briefly for 7 minutes at $37^{\circ} \mathrm{C}$ in an incubator to avoid any contamination of fibroblast or smooth muscle cells. The cords were massaged gently to facilitate cell detachment and were washed with M200 medium supplemented with an LSGS kit containing fetal bovine serum, $2 \% \mathrm{v} / \mathrm{v} ; 1 \mathrm{mg} / \mathrm{ml}$ hydrocortisone; $10 \mathrm{ng} / \mathrm{ml}$ human epidermal growth factor; $3 \mathrm{ng} /$ $\mathrm{ml}$ basic fibroblast growth factor; and $10 \mathrm{mg} / \mathrm{ml}$ heparin (Gibco, California, USA).

HUVECs were cultured in a 6-well plate (Nunc, Roskilde, Denmark) for approximately 6 days until reaching $80 \%$ confluency.

HUVECs was identified microscopically by their polygonal phenotype and by flow cytometry using CD34, VCAM-1 antibodies for positive markers and CD45 antibody for negative marker (BD Biosciences, USA) [19, 20].

\section{CELL CULTURES}

DPSC and HUVECs passages 2-4 were used for the experiments (Figure 1A-B). DPSCs were cultured in osteogenic medium consist of: DMEM medium supplemented with $10 \%$ FBS, $100 \mathrm{U} / \mathrm{ml}$ penicillin, $100 \mu \mathrm{g} / \mathrm{ml}$ streptomycin and $1.25 \mu \mathrm{g} / \mathrm{ml}$ Fungizone, $100 \mu \mathrm{g}$ ascorbic acid, and $10 \mathrm{mM} \beta$-glycerophosphate, $10 \mathrm{nM}$ dexamethasone. In the treatment group, DPSCs media were supplemented with 25\%, 50\%, 75\%, 100\% HUVECs-conditioned medium (day 5); DPSCs were co-cultured HUVECs with $1: 1 ; 1: 2 ; 1: 4$ HUVECs: DPSCs ratio in direct co-culture as well as indirect co-culture using a transwell insert system. Culture medium was collected at day 3, 5, 7 and the total protein was measured using Bradford total protein assay (Bio-Rad protein assay kit, Bio-Rad, USA). ALP assay was carried out using $10 \mu \mathrm{g}$ of protein added to diethanolamine buffer and P-nitrophenyl phosphate. The absorbance value was read at $405 \mathrm{~nm}$. Collagen type I protein and fibronectin protein were analyzed by ELISA assay. Briefly, a 96-well plate was incubated with 
A

The effect of HUVECs CM on

collagen I expression secreted by DPSCs

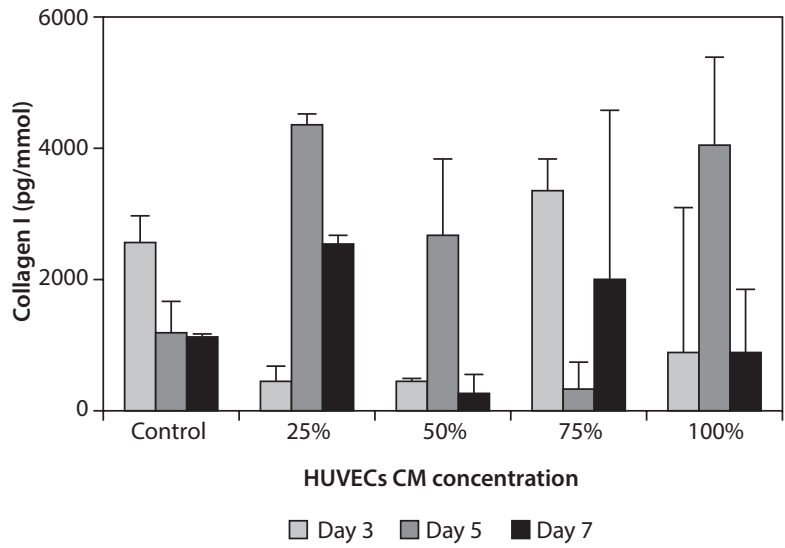

C $\begin{gathered}\text { The effect of HUVECs CM on } \\ \text { fibronectin secreted by DPSCs }\end{gathered}$

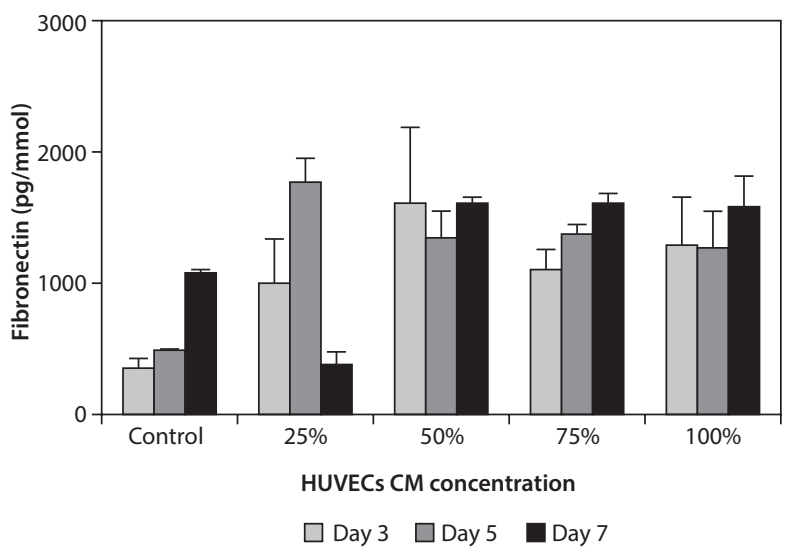

$\mathrm{E} \quad \begin{gathered}\text { The effect of HUVECs CM on } \\ \text { ALP activity of DPSCs cells }\end{gathered}$

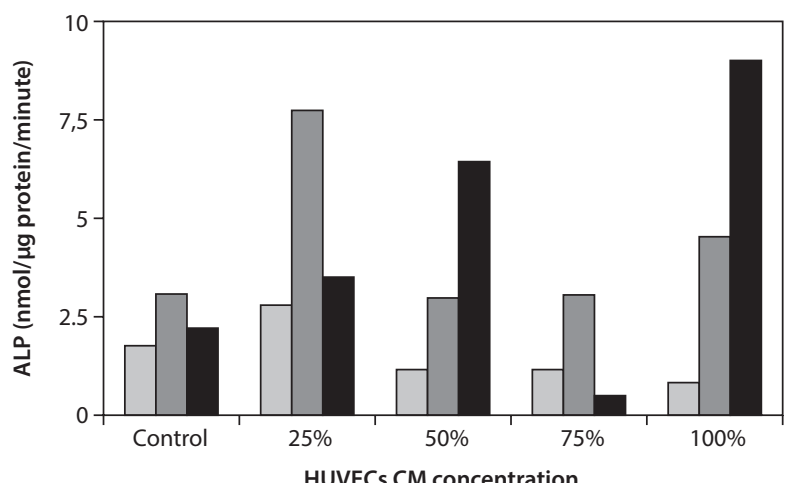

HUVECs CM concentration

$\square$ Day $3 \square$ Day 5 Day 7

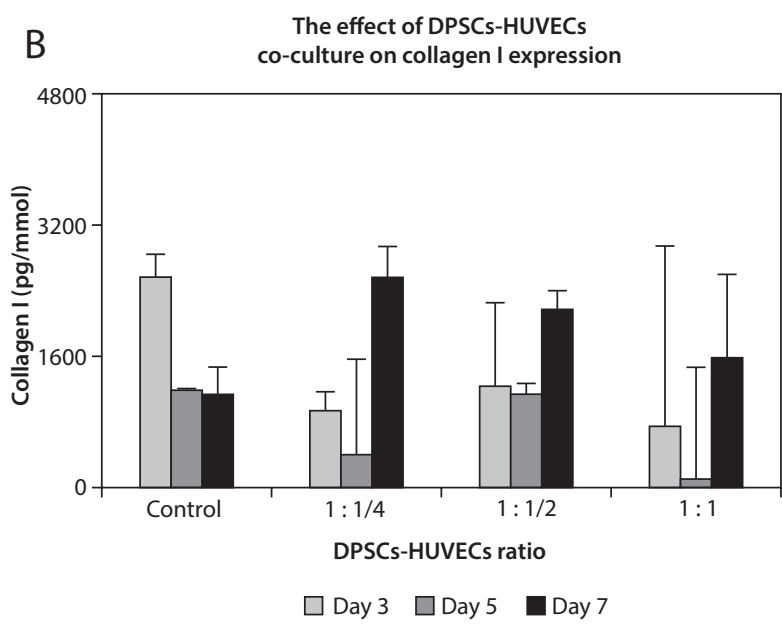

D The effect of DPSCs-HUVECs co-culture on fibronectin expression

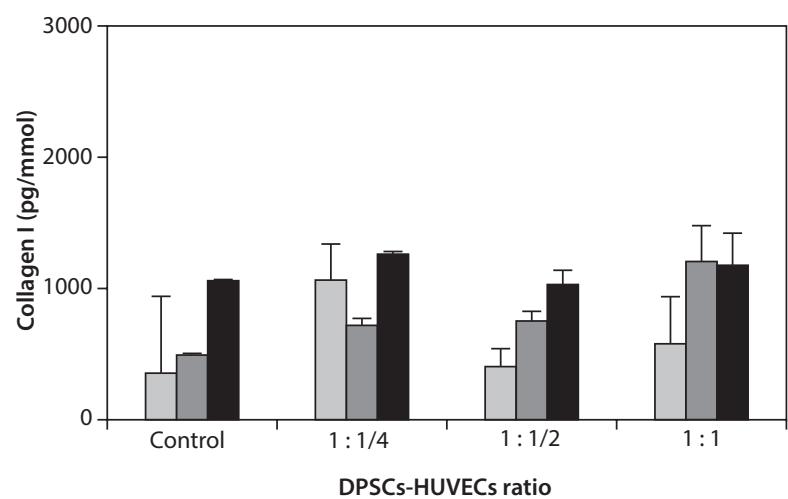

$\square$ Day $3 \quad \square$ Day $5 \square$ Day 7
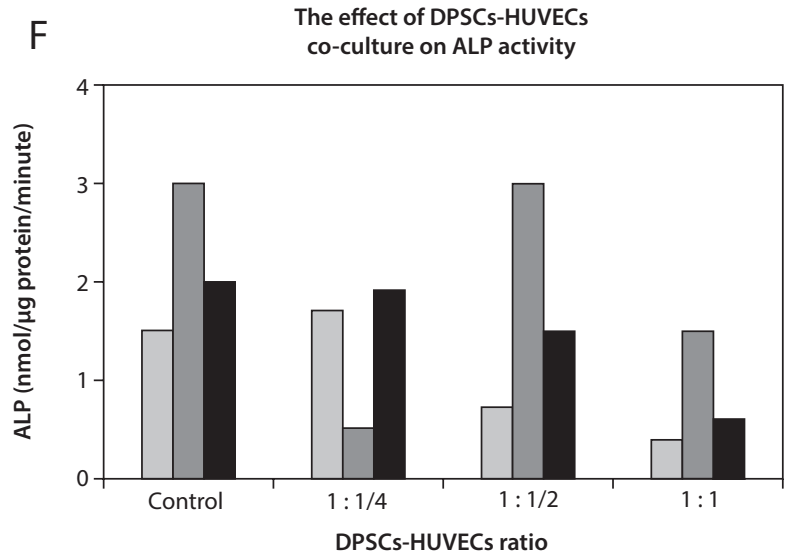

$\square$ Day $\quad \square$ Day 5 Day 7

FIGURE 2. The effect of human umbilical vein endothelial cells (HUVECS) conditioned medium (CM) on collagen I, fibronectin and alkaline phosphates (ALP) activity released by dental pulp cells (DPSCS) were shown in Figure $\mathbf{A}, \mathbf{C}, \mathbf{E}$. While the effect of DPSCS-HUVECs indirect co-culture on collagen I, fibronectin, ALP activity were shown in Figure $\mathbf{B}$, D, F, respectively

goat polyclonal to type 1 collagen dan goal polyclonal fibronectin overnight at $4^{\circ} \mathrm{C}$. The following day, the wells were washed with PBS 3 times and Neutravidin HRP $1: 20000$ in $1 \%$ BSA was added to the wells. The absorbance value was read at $405 \mathrm{~nm}$.

\section{STATISTICAL ANALYSIS}

GraphPad Prism 6 for Mac OS X was used to calculate data, which were presented as mean and standard deviation. Normality was tested using the Shapiro-Wilk nor- 
mality test. Data were analyzed using the Kruskal-Wallis test, and significance was accepted when $p<0.05$.

\section{RESULTS}

HUVECs-conditioned medium (CM) increased the expression of collagen I protein. The increase was seen at all $\mathrm{CM}$ concentrations tested with the highest found at CM $25 \%$ on day 5 . In contrast, collagen I decreased after DPSC-HUVECs co-culture for 3 and 5 days and started to increase on day 7. A similar observation was seen for the effect of CM on fibronectin proteins, where it increased the most at CM 25\% on day 5. The increase of fibronectin in the co-culture group was found on day 5 , earlier than the increase of collagen I found on day 7. The activity of ALP was measured and it showed the upregulation on days 5 and 7 after CM treatment while no upregulation of ALP was detected in the co-culture system at all time points tested (Figure 2).

\section{DISCUSSION}

Angiogenesis is an important process for the survival and differentiation of cells in bone tissue engineering [1]. A delay in angiogenesis may impair the survival of the cell-tissue construct in the defect area and could result in a less ideal clinical outcome. Endothelial cells play a role in the maintenance of vascular homeostasis as well as controlling the secretion of molecules that control cell proliferation $[2,3]$. Maintenance of angiogenesis in tissue engineering relies on the ingrowth of blood vessels from existing host vessels. As inadequate angiogenesis could disrupt the desired tissue regeneration, a potential strategy to compensate the limitation is to incorporate a growth factor-like compound that has the capacity to induce angiogenesis. In fact, many recent studies have proposed the potential of incorporating endothelial cells in a co-culture system to improve vascularization of the implanted cell-scaffold construct. Communication between endothelial and bone cells is crucial for controlling vascular supply during bone growth, remodeling, and repair. This study examined the interaction between HUVECs and human DPSCs. The effect of HUVECs on DPSCs differentiation towards an osteoblastic phenotype was studied by culturing them in two different types of HUVECs-DPSCs co-cultures, indirect contact and HUVECs-conditioned medium. Osteogenic markers of collagen I and ALP as well as fibronectin protein expression were evaluated at different time points. Our studies revealed that: (i) DPSCs responded earlier to HUVECs-conditioned medium compared to co-culture systems in terms of the secretion of collagen I and fibronectin; (ii) HUVECs-conditioned medium also promoted the ALP activity but not in HUVECs-DPSCs co-culture up to 7 days time point. These data highlight the potential of HUVECs-condi- tioned medium as a stimulus for osteogenic differentiation of DPSCs.

Previous studies reported the effect of direct and indirect HUVECs-DPSCs co-cultures, enhancing the odontogenic potential of DPSCs as well as vasculogenic capacity of HUVECs in cell-cell contact culture [13-15]. Higher alkaline phosphatase (ALP) activity level, mRNA expression of ALP, bone sialoprotein (BSP), dentin sialophosphoprotein (DSPP) genes and alizarin red staining were found in the co-culture group compared to the DPSCs alone. The study suggested that the higher odontogenic potential of DPSCs and vasculogenic capacity of HUVECs in the HUVECs-DPSCs co-culture system was facilitated by direct cell-cell contact, autocrine and paracrine stimulation. Our study tested the hypothesis that EC-conditioned medium has the capacity to promote DPSCs osteogenic differentiation as observed in the HUVECs-DPSCs co-culture system. Our study presented evidence that HUVECsconditioned medium has a better potential to induce osteogenic differentiation of DPSCs. The presence of signaling molecules in the HUVECs-conditioned medium stimulates the differentiation of DPSCs. Previous studies have demonstrated that human ECs produce soluble mediators that enhance human osteogenic behavior [6]. VEGF has a possible role as an indirect regulator of osteoblasts operating via endothelial cell-derived osteogenic factors that modify osteoblasts. VEGF promotes IGF-I and endothelin release from ECs to modify osteoblast differentiation [6].

The effect of endothelial cell-conditioned medium has been tested in bone marrow-derived mesenchymal stromal cells (BM-MSCs) [21]. In contrast to our finding, EC-conditioned medium inhibit BM-MSCs' differentiation into mature osteoblasts. BM-MSCs are the most utilized MSCs and their characteristics are well studied [22]. The characteristics of BM-MSCs and DPSCs share many similarities and differences. Growth curves and colony formation assay indicated a higher proliferation rate of DPSCs compared to BM-MSCs. BM-MSCs have more adipogenic potential and less osteogenic potential compared to DPSCs $[23,24]$. The differences in their characteristics might explain the contradictory results of BM-MSCs and DPSCs toward their differentiation. DPSCs provide a promising alternative source of MSCs for bone tissue engineering [25-27]. The conclusion of this study is that HUVECs-conditioned medium has the potential to be used to stimulate the differentiation of dental pulp cells to become osteoblasts, which is necessary for bone tissue engineering.

\section{CONFLICT OF INTEREST}

The authors declare no potential conflicts of interest with respect to the research, authorship, and/or publication of this article. 


\section{References}

1. Hankenson KD, Dishowitz M, Gray C, Schenker M. Angiogenesis in bone regeneration. Injury 2011; 42: 556-561.

2. Clarkin CE, Emery RJ, Pitsillides AA, Wheeler-Jones CP. Evaluation of VEGF-mediated signaling in primary human cells reveals a paracrine action for VEGF in osteoblast-mediated crosstalk to endothelial cells. J Cell Physiol 2008; 214: 537-544.

3. Guillotin B, Bareille R, Bourget C, et al. Interaction between human umbilical vein endothelial cells and human osteoprogenitors triggers pleiotropic effect that may support osteoblastic function. Bone 2008; 42: 1080-1091

4. Schipani E, Maes C, Carmeliet G, Semenza CL. Regulation of osteogenesis-angiogenesis coupling by HIFs and VEGF. J Bone Min Res 2009; 24: 1347-1353.

5. Grellier M, Ferreira-Tojais N, Bourget C, et al. Role of vascular endothelial growth factors in the communication between human osteoprogenitors and endothelial cells. J Cell Biochem 2009; 15: 390-398.

6. Street J, Lenehan B. Vascular endothelial growth factor regulates osteoblast survival-evidence for an autocrine feedback mechanism. J Orthop Surg Res 2009; 4: 19.

7. Awwad HK, el Naggar M, Mocktar N, Barsoum M. Intercapillary distance measurement as an indicator of hypoxia in carcinoma of the cervix uteri. Int J Radiat Oncol Biol Phys 1986; 12: 1329-1333.

8. Dohle E, Fuchs S, Kolbe M, et al. Sonic hedgehog promotes angiogenesis and osteogenesis in a coculture system consisting of primary osteoblasts and outgrowth endothelial cells. Tissue Eng Part A 2010; 16: 1235-1237.

9. Herzog DP, Dohle E, Bischoff I, Kirkpatrick CJ. Cell communication in a coculture system consisting of outgrowth endothelial cells and primary osteoblasts. Biomed Res Int 2014; 2014: 320123.

10. Grellier M, Bordenave L, Amédée J. Cell-to-cell communication between osteogenic and endothelial lineages: implications for tissue engineering. Trends Biotechnol 2009; 27: 562-571.

11. Yu H, VandeVord PJ, Mao L, et al. Improved tissue-engineered bone regeneration by endothelial cell mediated vascularization. Biomaterials 2009; 30: 508-517.

12. Gronthos S, Mankani M, Brahim J, et al. Postnatal human dental pulp stem cells (DPSCs) in vitro and in vivo. Proc Natl Acad Sci U S A 2000; 97: 13625-13630.

13. Dissanayaka, WL, Zhan X, et al. Coculture of dental pulp stem cells with endothelial cells enhances osteo-/odontogenic and angiogenic potential in vitro. J Endod 2012; 4: 454-463.

14. Gharaei MA, Xue Y, Mustafa K, et al. Human dental pulp stroma cell conditioned medium alters endothelial cell behavior. Stem Cell Res Ther 2018; 9: 69.

15. Pandula P, Samaranayake L, Jin L, Zhang, CF. Human umbilical vein endothelial cells synergize osteo/odontogenic differentiation of periodontal ligament stem cells in 3D cell sheets. J Periodont Res 2014; 49: 299-306.

16. Zhang C, Chang J, Sonoyama W, et al. Inhibition of human dental pulp stem cell differentiation by Notch signaling. J Dent Res 2008 87: 250-255.

17. Kaigler D, Krebsbach PH, West ER, et al. Endothelial cell modulation of bone marrow stromal cell osteogenic potential. FASEB J 2005; 19: 665-667.

18. Amir LR, Suniarti DF, Utami S, Abbas B. Chitosan as a potential osteogenic factor compared with dexamethasone in cultured macaque dental pulp stromal cells. Cell Tissue Res 2014; 358: 407-415.

19. Amir LR, Bachtiar EW, Puspitawati R, et al. Increased endothelial cell growth in culture supplemented with outdated human platelet lysate. J Int Dent Med Res 2016; 9 (Special Issue): 368-375.

20. Amir LR, Puspitawati R, Hazriani R, et al. No recombinant EGF and bFGF is required on HUVECs culture supplemented with human platelet lysate. J Int Dent Med Res 2017; 10: 3 .

21. Saleh FA, Whyte M, Ashton P, et al. Regulation of mesenchymal stem cell activity by endothelial cells. Stem Cells Dev 2011; 20: 391-403.
22. Xu L, Liu Y, Sun Y, et al. Tissue source determines the differentiation potentials of mesenchymal stem cells: a comparative study of human mesenchymal stem cells from bone marrow and adipose tissue. Stem Cell Res Ther 2017; 8: 275.

23. Duffy GP, Ahsan T, O'Brien T, et al. Bone marrow-derived mesenchymal stem cells promote angiogenic processes in a time- and dose-dependent manner in vitro. Tissue Eng Part A 2009; 15: 2459-2470.

24. ShiS, Gronthos S. Perivascular niche of postnatal mesenchymal stem cells in human bone marrow and dental pulp. J Bone Miner Res 2003; 18: 696-704.

25. Nakashima M, Iohara K. Regeneration of dental pulp by stem cells. Adv Dent Res 2011; 23: 313-319.

26. Huang GT. Dental pulp and dentin tissue engineering and regeneration: advancement and challenge. Front Biosci (Elite Ed) 2011; 3: 788-800.

27. Bronckaers A, Hilkens P, Fanton Y, et al. Angiogenic properties of human dental pulp stem cells. PLoS One 2013; 8: e71104. 